\title{
Exploiting the airwave for time-lapse reservoir monitoring with CSEM on land
}

\author{
Marwan Wirianto ${ }^{1}$, Wim A. Mulder ${ }^{2}$, and Evert C. Slob ${ }^{1}$
}

\begin{abstract}
In the application of controlled source electromagnetics for reservoir monitoring on land, repeatability errors in the source will mask the time-lapse signal due to hydrocarbon production when recording surface data close to the source. We demonstrate that at larger distances, the airwave will still provide sufficient illumination of the target. The primary airwave diffuses downward into the earth and then is scattered back to the surface. The time-lapse difference of its recorded signal reveals the outline on the surface of the resistivity changes in a hydrocarbon reservoir under production. However, repeatability errors in the primary airwave can destroy the signal-to-noise ratio of the time-lapse data. We present a simple and effective method to remove the primary airwave from the data, which we call partial
\end{abstract}

airwave removal. For a homogeneous half space and a deltafunction type of source, the surface expression of the airwave does not depend on frequency. For this reason, the primary airwave can be subtracted from the data using recordings at two frequencies, one low enough with a skin depth of the order of the reservoir depth that is sensitive to the reservoir, the other high enough to only sense the near surface. The method does not affect secondary airwave components created by signals that have propagated through the earth and returned to the surface. We show that the method provides a direct indicator of production-related time-lapse changes in the reservoir. We illustrate this for several models, including a general 3D heterogeneous model and one with strong surface topography, for situations where survey repeatability errors are large.

\section{INTRODUCTION}

During the last decade, controlled source electromagnetics (CSEM) has been a subject of continued interest in the oil and gas industry. Early enthusiasm was triggered by the success of a pilot CSEM experiment for detecting thin resistive hydrocarbon reservoirs located offshore Angola (Ellingsrud et al., 2002). Since then, several authors have reported and established its position as a tool for derisking potential hydrocarbon prospects next to seismics; see, for example, the March-April 2007 issue of Geophysics, the March 2007 issue of The Leading Edge, and the May 2009 and May 2010 issues of First Break. Another potential application of CSEM is hydrocarbon reservoir monitoring during production. Water flooding or steam injection for oil production creates resistivity changes in the reservoir, and those changes can potentially be detected with time-lapse CSEM measurements. Several feasibility studies on time-lapse CSEM
(Lien and Mannseth, 2008; Orange et al., 2009; Wirianto et al., 2009, 2010a; MacGregor and Cooper, 2010) led to optimistic conclusions. Earlier, Wright et al. (2002) obtained positive results with field data when monitoring gas storage in a shallow, 25-m-thick reservoir containing gas and water.

In our own synthetic feasibility study (Wirianto et al., 2010a), we considered land CSEM for a deeper target with oil and brine in a complex 3D resistivity model. We studied the effect of additive and multiplicative noise and repeatability errors on the time-lapse electromagnetic signal. Close to the source, multiplicative repeatability errors of the direct field completely drown the time-lapse measurements. Farther away, surface time-lapse data reveal the outlines of the subsurface resistivity changes, assuming oil depletion of a rather large reservoir volume. With a smaller depleted volume, the production-related time-lapse signal becomes harder to distinguish, especially in the presence of repeatability errors (Wirianto et al., 2010b).

Manuscript received by the Editor 20 September 2010; revised manuscript received 27 November 2010; published online 12 April 2011.

${ }^{1}$ Delft University of Technology, Delft, The Netherlands. E-mail: marwan_wirianto@yahoo.com; m.liam@tudelft.nl; e.c.slob@tudelft.nl.

${ }^{2}$ Delft University of Technology, Delft, The Netherlands and Shell Global Solutions International, Rijswijk, The Netherlands. E-mail: wim.mulder @shell.com.

(C) 2011 Society of Exploration Geophysicists. All rights reserved. 


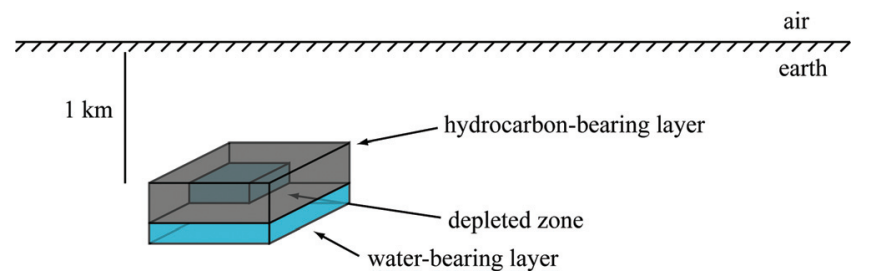

Figure 1. Geometry of a simple monitoring problem.

a)
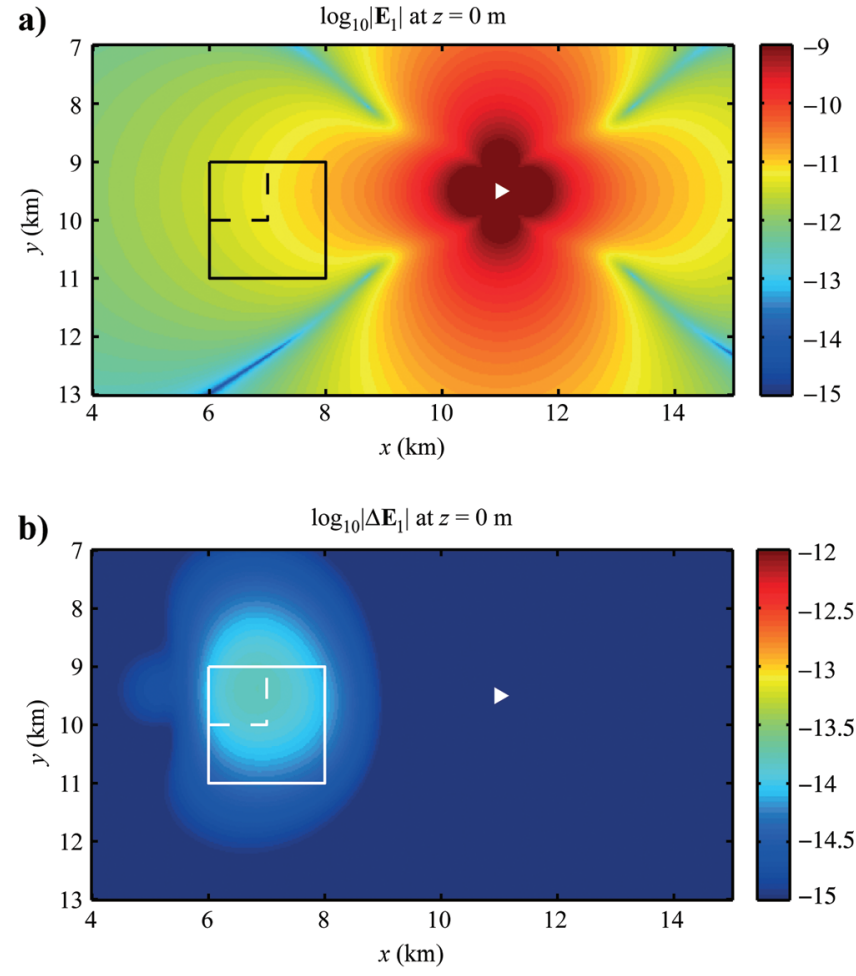

c)

c) $\log _{10}\left|\Delta \mathbf{E}_{1}\right|$ at $z=0 \mathrm{~m}$, added noise

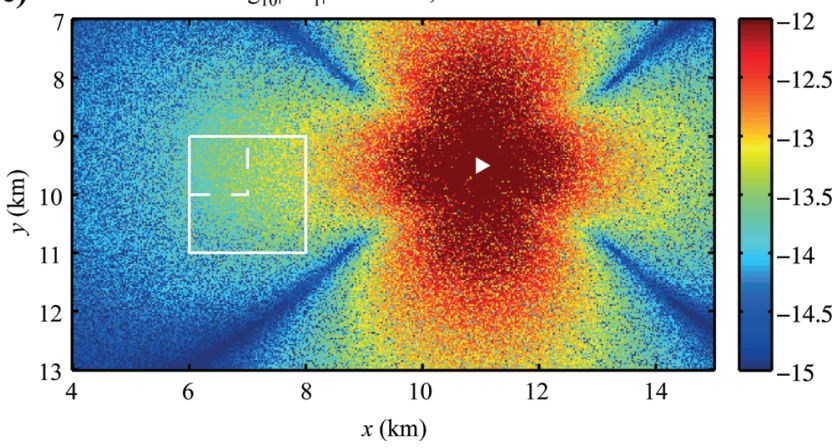

Figure 2. The electric field in the $x$-direction recorded at the surface in top view at $1 \mathrm{~Hz}$. (a) The total field before production. (b) The time-lapse difference without noise. (c) The timelapse difference with noise. In each panel, the solid box indicates the lateral position of the reservoir, and the dashed line indicates the lateral position of the oil-water interface after flooding. The white triangle marks the lateral location of the HED source.
This led to two questions: why can we still observe timelapse signals at a larger distance from the source, and how can we improve their signal-to-noise ratio $(\mathrm{S} / \mathrm{N})$ ? In the following section, we explain that the primary airwave provides the answer to the first question. It is the dominant signal at the surface and, when diffused into the earth, the main source of illumination for the reservoir. However, repeatability errors will affect the primary airwave, just as happens with the direct field, and these can mask the time-lapse signal from the swept reservoir region. To improve its $\mathrm{S} / \mathrm{N}$, we have to remove the primary airwave from the recorded data, while keeping that part of the airwave that has diffused into the earth and then back to the surface and into the air. For sources and receivers on the surface, we found an effective way to accomplish what we call partial airwave removal, which removes only the primary component that travels directly from source to receiver and therefore also gets rid of its related repeatability errors. We present numerical examples that test whether this idea still works in the presence of near-surface heterogeneities or significant surface topography.

\section{THE AIRWAVE}

We will explain the impact of the airwave on the monitoring problem with a simple example, assuming a flat horizontal surface and a homogeneous half space. Figure 1 sketches the configuration of a land CSEM monitoring problem. We consider a reservoir under production in a $2 \Omega \mathrm{m}$ homogeneous half space. The reservoir has dimensions of 2 by 2 by $0.5 \mathrm{~km}^{3}$ and consists of a hydrocarbon-bearing part and a water-bearing part. The hydrocarbon-bearing part has a resistivity of $100 \Omega \mathrm{m}$ and is a $300-\mathrm{m}$-thick layer at a depth of $1 \mathrm{~km}$ below the air-earth interface. Below it, a 200-m-thick water-bearing layer has a resistivity of $3 \Omega \mathrm{m}$. For the monitoring study, we assume the reservoir is flooded by saline water from the top left, creating a small, 100-m-thick, box-shaped depleted zone in the corner of the hydrocarbon-bearing layer. Moreover, we also assume that the CSEM measurements are available in the frequency domain. We computed the electric field response for this time-lapse configuration with a multigrid solver (Mulder, 2006).

Figure 2 displays the amplitude behavior of the time-lapse difference for the $x$-directed electric field measured on the surface, excited by a unit $x$-directed horizontal electric dipole (HED) point source located at $(11,9.5,0) \mathrm{km}$ and operating at $1 \mathrm{~Hz}$. The time-lapse difference shown in Figure $2 \mathrm{~b}$ is laterally confined to the location of resistivity change. In practice, we will not obtain such a nice result because repeatability errors will affect the time-lapse measurements. These will have instrumental as well as natural causes (for instance, frost) and will appear as multiplicative noise in the recorded data before and after production. As an illustration, we use the same approach as Wirianto et al. (2010a), mimicking repeatability errors by adding random numbers to the measured electric fields with a maximum amplitude of $1 \%$ relative to the signal strength at each receiver. Figure $2 \mathrm{c}$ shows that this noise imposes a strong source imprint and completely masks the time-lapse signal. We therefore have to better understand what is going on when we try to detect the time-lapse resistivity changes in the reservoir. 
The depleted volume can be considered as a scattering object. To first order, the time-lapse difference is the scattered field, contaminated by repeatability errors in the total field. The behavior of the secondary fields is determined by the extent, depth, and resistivity contrast of the depleted zone, as well as the background signals that are excited by the source (Tehrani and Slob, 2010, e.g.). Because the extent and resistivity contrast of the depleted zone represent an existing situation, the detectability of the time-lapse change depends primarily on the incoming fields at the depleted zone. For the model shown in Figure 1, we can simply use the solution for a homogeneous half-space (Raiche and Coggon, 1975), ignoring higher-order scattering inside the reservoir. Figure 3 shows a vertical crosssection through the source of the incoming electric field excited by an $x$-directed HED point source. The color shows the amplitude of the electric field on a logarithmic scale. The black arrows follow the current. Apart from the direct field of the source, there is a significant contribution at shallow depths from the airwave, which is the electromagnetic field that propagates in the air with the speed of light. The part that propagates along the surface is called a lateral wave. It sends a field into the ground with an almost vertical diffusion direction (Baños, 1966).

Generally, with an HED source on the surface, the EM fields are composed of two types, the direct field and the airwave, which can be separated following Slob et al. (2010). The airwave amplitude is proportional to inverse horizontal distance to the power of 3 , whereas it shows an exponential decay as a function of depth. The direct field diffuses through the ground and shows an exponential decay as a function of distance. Separate analysis of each contribution shows that the airwave dominates for offsets exceeding $2 \mathrm{~km}$, both on the surface and at the depth of the depleted volume. From this analysis it follows that the airwave provides the largest contribution to the time-lapse signal if the source is not too nearby. The fact that the airwave is also the predominant signal on the surface will create large multiplicative repeatability errors in the time-lapse measurements. Let us look at this in more detail. With time-lapse measurements, we collect two data sets, before and after production. The latter can be expressed as $\mathbf{E}^{d}+\mathbf{E}^{a w}+\mathbf{E}^{d ; s c}+\mathbf{E}^{a w ; s c}$. The first two terms denote the incident fields, with the direct field and the primary airwave contribution, whereas the last two terms denote the scattered fields representing the time-lapse change. If we assume this change is entirely due to the resistivity change in the reservoir, the component $\mathbf{E}^{d}+\mathbf{E}^{\text {aw }}$ will cancel out when considering the measurements at different times. In the presence of multiplicative noise, however, the difference becomes $\alpha\left(\mathbf{E}^{d}+\mathbf{E}^{a w}\right)+\mathbf{E}^{d ; s c}+\mathbf{E}^{a w ; s c}$, where the factor $\alpha$ models the multiplicative repeatability errors. For $\mid \mathbf{E}^{d ; s c}$ $+\mathbf{E}^{a w ; s c}|\ll| \alpha\left(\mathbf{E}^{d}+\mathbf{E}^{a w}\right) \mid$, this noise becomes a problem, as illustrated in Figure 2c.

From these results, we conclude that the airwave can be beneficial if we can reduce or remove the contribution $\mathbf{E}^{\text {aw }}$ due to the primary airwave generated by the source, while maintaining the part of the airwave $\mathbf{E}^{a w ; s c}$ that is transmitted into the ground, has a large enough amplitude at reservoir depth, and can produce a significant time-lapse response. This leads us to the concept of partial airwave removal.

\section{PARTIAL AIRWAVE REMOVAL}

In the time domain, the airwave related to a delta-function source at the surface shows up as an instantaneous delta spike, which can be surgically removed from the data. Because the Fourier transform of the delta function has a flat spectrum, we propose to perform partial airwave removal in the frequency domain by comparing CSEM measurements at two distinct frequencies. This approach remotely resembles the method proposed by Maaø and Nguyen (2010) but is essentially different. Their frequency differencing amounts to data weighting with a factor proportional to time, with the purpose of boosting later arrivals that have seen deeper structures. Noise is boosted as well, so the method requires data with a good S/N. For time-lapse measurements, we only want to remove the primary airwave from the measurements to improve the $\mathrm{S} / \mathrm{N}$ of the time-lapse signal. We therefore select two frequencies that are sufficiently far apart. One should be low enough to have a skin depth of the order of the target depth, so that it sees the reservoir. The other should be high enough to sense only the near surface. Subtracting the two will emphasize the signal from the deeper target and remove the repeatability errors from the primary airwave.

We will illustrate the method by numerical examples. As a first test, we applied partial airwave removal to the earlier monitoring example. Figure 4 displays the results. Figure $4 \mathrm{a}$ shows the time-lapse difference at $1 \mathrm{~Hz}$ in the presence of multiplicative noise before applying the removal. For Figure $4 \mathrm{~b}$, we first subtracted the measured responses at $10 \mathrm{~Hz}$ and then computed the time-lapse difference. Now, the lateral extent of the depleted zone is better defined, even in the presence of repeatability error.

In the second test, we considered the effect of topography on the airwave. Figure 5 shows the elevation model, with the greatest difference in height being $1 \mathrm{~km}$. The reservoir is located 1 $\mathrm{km}$ below the hilly area, whereas the source is placed in the valley, $600 \mathrm{~m}$ below the highest point, as indicated by a white star. Figure 6 shows the $x$-component of the electric field measured on the surface. Figure $6 \mathrm{a}$ shows the time-lapse difference at 1
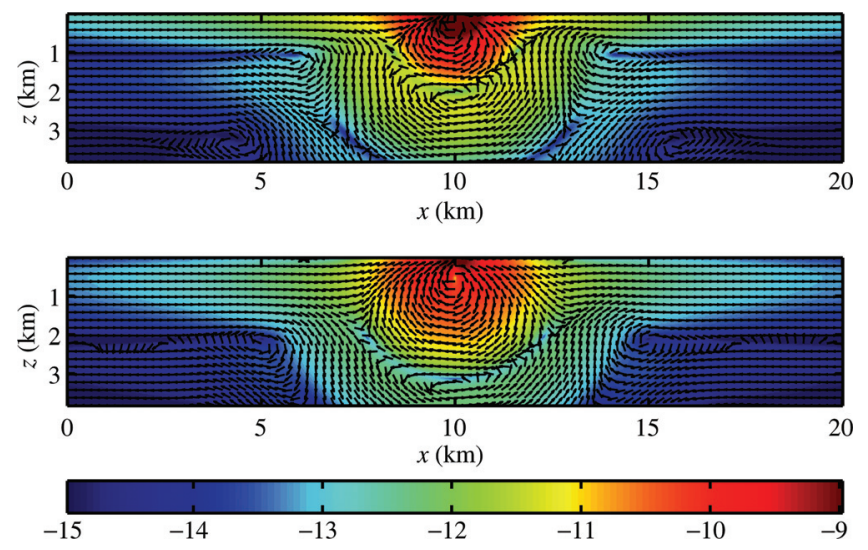

Figure 3. Electric-field vector plots in a vertical section of a $2 \Omega \mathrm{m}$ half-space model excited by an $x$-directed HED point source. The source is located at $(10,0,0) \mathrm{km}$, just below the airearth interface, and operates at $1 \mathrm{~Hz}$. The top panel displays the real part of the electric field (the in-phase mode), whereas the bottom panel shows its imaginary part (the out-of-phase mode). 
$\mathrm{Hz}$ without partial airwave removal, and Figure $6 \mathrm{~b}$ shows it with partial airwave removal, using the response at $10 \mathrm{~Hz}$. Clearly, after removal, the lateral extent of the depleted zone is better defined.

The third test involves a heterogeneous background but a flat surface. We used the same model as in Wirianto et al. (2010a) with a slight modification. Instead of vertical, piston-like displacement, we assumed that the oil production affects only the small area shown in Figure 7. A unit dipole source in the $x$-direction was positioned on the surface at $(x, y)=(12,9.5) \mathrm{km}$. The frequency

a)
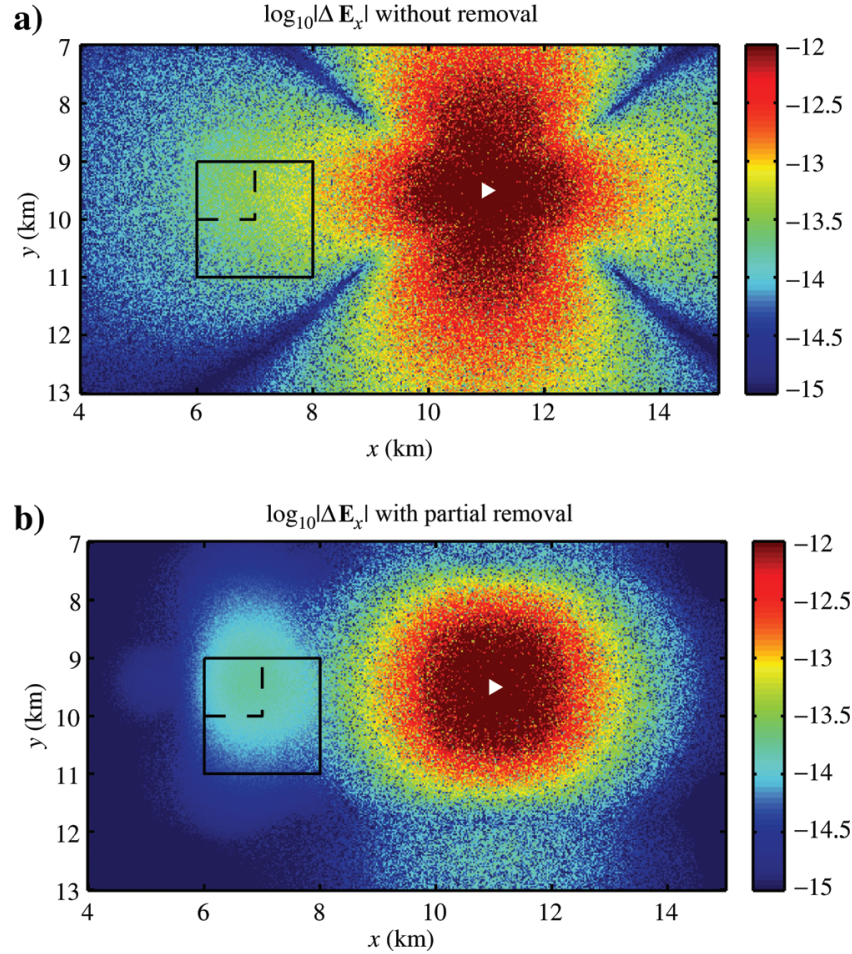

Figure 4. (a) The time-lapse difference of the electric field in the $x$-direction recorded at the surface in top view at $1 \mathrm{~Hz}$, as in Figure 2c. (b) The result after partial airwave removal.

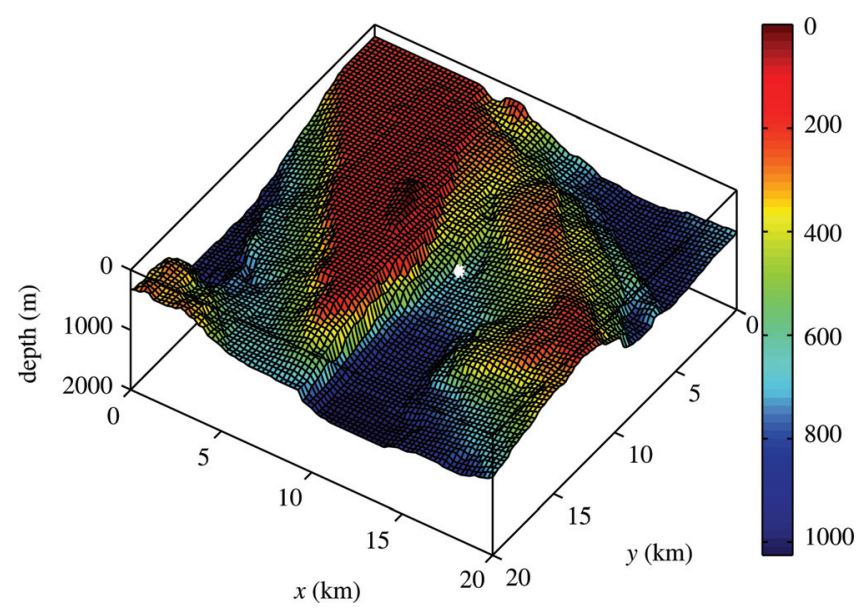

Figure 5. Topography model. was set to $5 \mathrm{~Hz}$. Figure 8 a shows the time-lapse difference of an $x$ directed electric field measured on the surface without partial airwave removal, and Figure $8 \mathrm{~b}$ shows it with partial airwave removal, using the EM field at $10 \mathrm{~Hz}$. The results show that the method also performs well with a heterogeneous resistivity model. Again, the lateral extent of the depleted zone is better defined, even in the presence of repeatability errors.
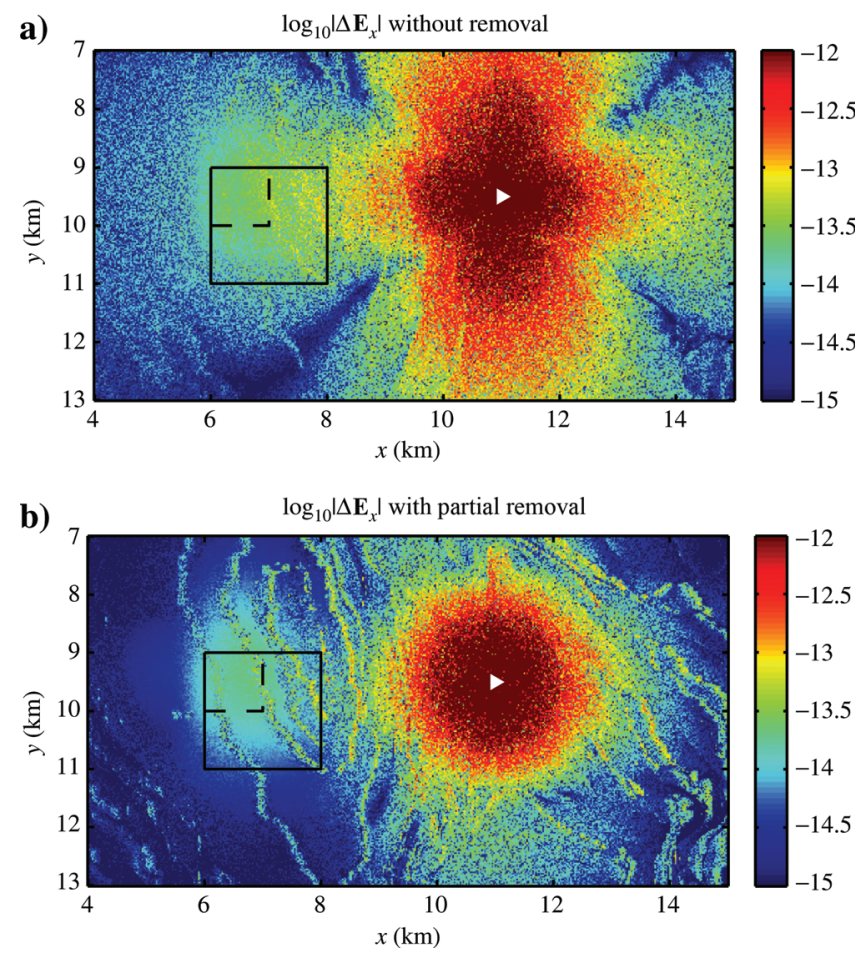

Figure 6. As described in Figure 4, but with topography.

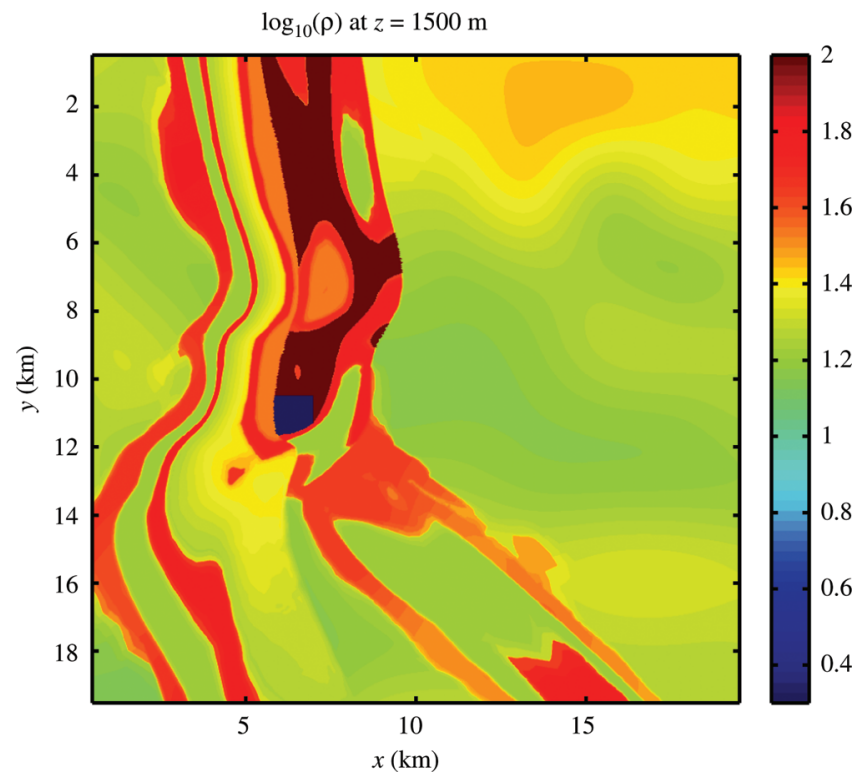

Figure 7. The horizontal slice of the model at the depth of the depleted volume. Dark blue indicates the depleted volume. 
a)
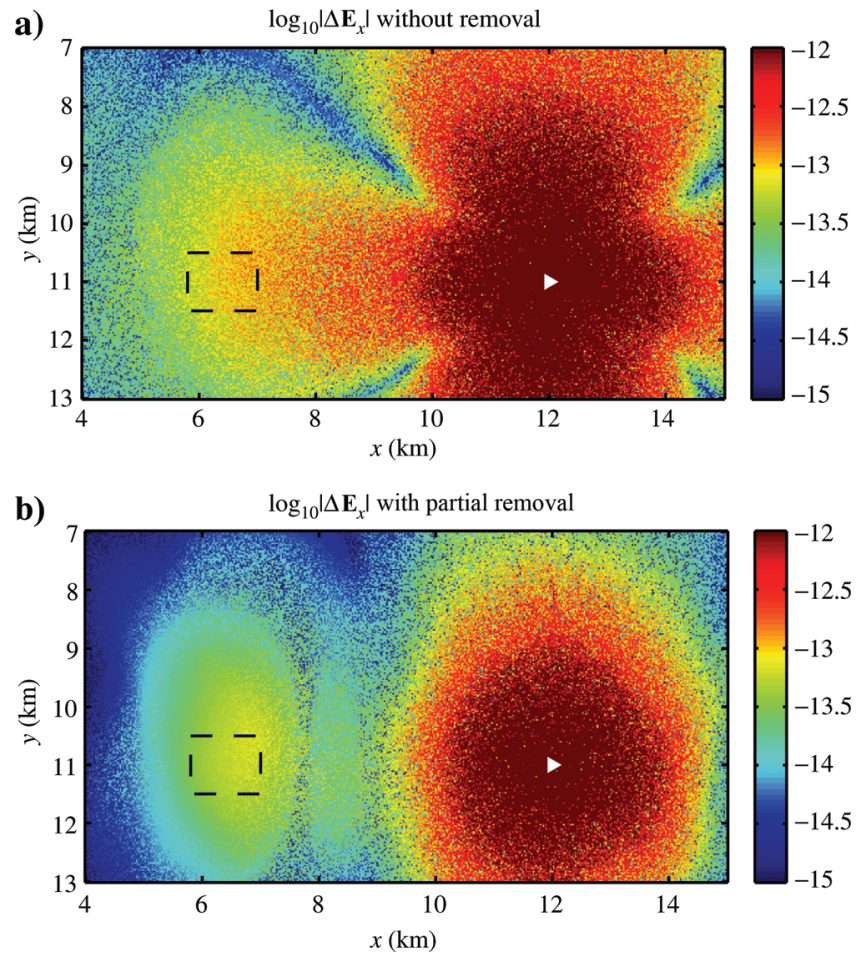

Figure 8. Panels (a) and (b) are similar to Figure 4, but for a heterogeneous background. We applied a frequency of $5 \mathrm{~Hz}$ to observe the target and $10 \mathrm{~Hz}$ to model the airwave effect on the near surface. In each panel, the dashed black line indicates the lateral position of the oil-water interface after flooding. The small white triangle marks the lateral location of the HED source.

\section{CONCLUSIONS}

The airwave can produce a better time-lapse signal in the presence of repeatability noise than the direct field from the source, because we are able to remove the primary airwave component. This is common practice in time-domain measurements. We proposed a similar approach for frequency-domain measurements. Subtracting a simultaneous measurement of data at a higher frequency from a measurement at a lower frequency that has a large enough skin depth to reach the reservoir removes the airwave and its related multiplicative noise from the time-lapse data.

Numerical experiments demonstrated a substantial improvement of the signal-to-noise ratio of the time-lapse signal after partial airwave removal, not only in a homogeneous background model, but also with topography or strong heterogeneities. Our results indicate that the concept of partial airwave removal potentially opens up a new avenue for land CSEM reservoir monitoring using frequency-domain systems. One of the remaining issues is the optimal choice of the pair of frequencies for partial airwave removal. Another is its practical use in a field test.

\section{ACKNOWLEDGMENTS}

Marwan Wirianto received financial support from the sponsors of the Delphi Consortium.

\section{REFERENCES}

Baños, A., 1966, Dipole radiation in the presence of a conducting halfspace: Pergamon Press, Inc.

Ellingsrud, S., T. Eidesmo, S. Johansen, M. C. Sinha, L. M. MacGregor, and S. Constable, 2002, Remote sensing of hydrocarbon layers by seabed logging (SBL): Results from a cruise offshore Angola: The Leading Edge, 21, 972-982, doi:10.1190/1.1518433.

Lien, M., and T. Mannseth, 2008, Sensitivity study of marine CSEM data for reservoir production monitoring: Geophysics, 73, no. 4, F151-F163, doi:10.1190/1.2938512

Maaø, F. M., and A. K. Nguyen, 2010, Enhanced subsurface response for marine CSEM surveying: Geophysics, 75, A7-A10, doi:10.1190/ 1.3377054

MacGregor, L. M., and R. Cooper, 2010, Unlocking the value of CSEM: First Break, 28, 49-52.

Mulder, W. A., 2006, A multigrid solver for 3D electromagnetic diffusion: Geophysical Prospecting, 54, no. 5, 633-649, doi:10.1111/j.13652478.2006.00558.x

Orange, A., K. Key, and S. Constable, 2009, The feasibility of reservoir monitoring using time-lapse marine CSEM: Geophysics, 74, no. 2, F21-F29, doi:10.1190/1.3059600.

Raiche, A. P., and J. H. Coggon, 1975, Analytic Green's tensors for integral equation modelling: Geophysical Journal of the Royal Astronomical Society, 42, no. 3, 1035-1038, doi:10.1111/j.1365246X.1975.tb06465.x.

Slob, E., J. Hunziker, and W. Mulder, 2010, Green's tensors for the diffusive electric field in a VTI half-space: Progress in Electromagnetics Research, 107, 1-20, doi:10.2528/PIER10052807.

Tehrani, A. M., and E. Slob, 2010, Fast and accurate three-dimensional controlled source electro-magnetic modelling: Geophysical Prospecting, 58, 1133-1146.

Wirianto, M., W. A. Mulder, and E. C. Slob, 2009, A study of land CSEM reservoir monitoring in a complex 3D model: 71st Annual International Conference \& Exhibition, EAGE, Extended Abstracts, X020.

, 2010a, A feasibility study of land CSEM reservoir monitoring in a complex 3-D model: Geophysical Journal International, 181, 741755 .

, 2010b, A feasibility study of land CSEM reservoir monitoring: the effect of the airwave: Progress in Electromagnetic Research Symposium Online, 6, 440-444.

Wright, D., A. Ziolkowski, and B. Hobbs, 2002, Hydrocarbon detection and monitoring with a multicomponent transient electromagnetic (MTEM) survey: The Leading Edge, 21, 852-864, doi:10.1190/ 1.1508954 . 